\section{M.A. Musen', J.H. van Bemmel ${ }^{2}$}

${ }^{1}$ Stanford Medical Informatics

Stanford University School of Medicine

Stanford, California, USA

${ }^{2}$ Institute of Medical Informatics,

Erasmus University Medical Center,

Rotterdam, The Netherlands

\title{
Challenges in Medical Informatics
}

\section{A Discipline Coming of Age}

These are tough times in which to be an adolescent. Many health-care institutions are beginning to question the value of the investments that they have made in informatics. Some of our best scientists are leaving their university positions and many of our trainees seem ill prepared to take on academic jobs. Funding organizations are suddenly enraptured by the glamour of computational biology (which they confuse with bioinformatics), heightening the competition for limited resources. In these demanding times, workers in medical informatics do not have the luxury to act as teenagers and to brood about why the world may be treating them unfairly; they need to define their purpose clearly and to set achievable goals for the years ahead.

As we reported last year in these pages [1], a group of senior academicians in medical informatics convened in Madrid in March 2001 in conjunction with the meeting of the IMIA Board. The goal of the Madrid workshop was to define the challenges faced by medical informatics as an academic discipline and to suggest strategies to enhance the scholarly foundation of our field. Some of the position papers that were contributed to the Madrid workshop appeared last year in a special issue of Methods of Information in Medicine [2]. Four of those papers are now reprinted in this edition of the Yearbook. These papers were selected because they provide a crosssection of opinions and are representative of all the contributions discussed at the workshop.

The papers point to the need for our community to define clearly what medical informatics is and why it is important. We cannot continue to characterizemedicalinformaticsinterms of other, more established disciplines. If we claim that medical informatics is a kind of "applied computer science," for example, then it remains unclear why medical informatics-at least academically—needs to existapartfrom more traditional computer-science groups. If we claim that our field is simply an amalgam of other disciplines, such as computer science, biostatistics, health-services research, and cognitive science, then there is nothing that we can claim to be our own. Like teenagers struggling to understand what differentiates themselves from their parents, the participants at the Madrid workshop worked hard to characterize what are the unique elements of 
medical informatics that are distinct from those of the allied disciplines that contribute to our scientific enterprise.

Although it may seem obvious in retrospect, the universal conclusion from the Madrid workshop was that academic informatics is special because of its focus on information. Unlike computer science, for example, which typically places its emphasis on computation, our research community is dedicated to the study of information as a first-class object. It is modeling the data and knowledge required by our applications that requires unique skills, and where our academic research makes its distinguishing contribution. Ours is indeed the discipline that cares about the content. Our principal challenge in medical or health informatics is to understand better the structure of data, information, and knowledge, and to cast our scholarship in terms of appropriate models of these abstract entities.

Models of information, of course, lack cogency unless there are processes that operate on those models to perform useful tasks. Such tasks must be performed within complex social systems and must inform discerning and yet fallible human participants. Thus, it is impossible to consider information completely in isolation. Work in medical informatics is inherently interdisciplinary because of the need to draw on a large number of related fields that allow us to put the study of information into context. We are challenged to maintain our bridges to diverse, related fields such as cognitive science, image processing, and epidemiology, and to clarify the common foundation that we share with research in bioinformatics.

The participants at the Madrid workshop concluded their discussions articulating an urgent need to disseminate the perspective that informatics is both a science and an engineering discipline with a strong theoretical foundation. They saw a requirement to enhance our curricula to clarify the contributions of basic research in informatics-helping students to understand the underlying principles that transcend particular application domains and that provide coherency to our research programs. There also was a perception that professional societies, such as IMIA, must take the lead in educating both their members and funding bodies about the generalizable contributions of basic scholarship in informatics, and how a wide range of application areas - from health care to biology to other information-intensive activities — can benefit from academic research in informatics.
The papers reprinted in this edition of the Yearbook are a step toward the dissemination of the ideas discussed at the Madrid workshop. We hope that they will stimulate both continued introspection and a plan for action within our community as the field of medical informatics continues to come of age.

\section{References}

1. Musen, MA and Van Bemmel, JH. Challenges for medical informatics as an academic discipline: workshop report. In: Haux R and Kulikowski C, editors. Yearbook of Medical Informatics 2002. Stuttgart: Schattauer; 2002. p. 194-7.

2. Musen, MA and Van Bemmel, JH. editors. Special issue on Challenges for Medical Informatics as an Academic Discpline. Methods Inf Med 2002; 41:1-63.

Address of the authors:

Mark A. Musen

Stanford Medical Informatics

Stanford University School of Medicine

251 Campus Drive, Room X-215

Stanford, CA 94305-5479

USA

E-mail: Musen@SMI.Stanford.EDU

Jan H. van Bemmel

Institute of Medical Informatics

Erasmus University Medical Center

P.O. Box 1738

3000 DR Rotterdam

The Netherlands

E-mail: vanbemmel@mi.fgg.eur.nl 